\title{
Development/Plasticity/Repair
}

\section{Cholinergic Modulation of Binocular Vision}

\author{
Yasha Sheynin, ${ }^{1}{ }^{\circledR}$ Pedro Rosa-Neto, ${ }^{3}$ Robert F. Hess, ${ }^{1 \star}$ and ${ }^{\circledR}$ Elvire Vaucher ${ }^{2 \star}$ \\ ${ }^{1}$ McGill Vision Research, McGill University, Montréal, Quebec H3G 1A4, Canada, ${ }^{2}$ Laboratoire de Neurobiologie de la Cognition Visuelle, École \\ d'Optométrie, Université de Montréal, Montréal, Quebec H3T 1P1, Canada, and ${ }^{3}$ Douglas Mental Health University Institute, McGill University, \\ Montréal, Quebec H4H 1R3, Canada
}

\begin{abstract}
The endogenous neurotransmitter acetylcholine (ACh) is known to affect the excitatory/inhibitory (E/I) balance of primate visual cortex, enhancing feedforward thalamocortical gain while suppressing corticocortical synapses. Recent advances in the study of the human visual system suggest that $\mathrm{ACh}$ is a likely component underlying interocular interactions. However, our understanding of its precise role in binocular processes is currently lacking. Here we use binocular rivalry as a probe of interocular dynamics to determine ACh's effects, via the acetylcholinesterase inhibitor (AChEI) donepezil, on the binocular visual system. A total of 23 subjects (13 male) completed two crossover experimental sessions where binocular rivalry measurements were obtained before and after taking either donepezil $(5 \mathrm{mg}$ ) or a placebo (lactose) pill. We report that enhanced cholinergic potentiation attenuates perceptual suppression during binocular rivalry, reducing the overall rate of interocular competition while enhancing the visibility of superimposition mixed percepts. Considering recent evidence that perceptual suppression during binocular rivalry is causally modulated by the inhibitory neurotransmitter GABA, our results suggest that cholinergic activity counteracts the effect of GABA with regards to interocular dynamics and may modulate the inhibitory drive within the visual cortex.
\end{abstract}

Key words: acetylcholinesterase inhibitors; binocular rivalry; binocular vision; cholinergic modulation; excitatory/inhibitory balance; visual cortex

\section{Significance Statement}

Our research demonstrates that the cholinergic system is implicated in modulating binocular interactions in the human visual cortex. Potentiating the transmission of acetylcholine (ACh) via the cholinergic drug donepezil reduces the extent to which the eyes compete for perceptual dominance when presented two separate, incongruent images.

\section{Introduction}

Binocularity, a defining feature of human vision that enables stereopsis, is predicated on the ability to combine inputs from the two eyes to create a singular representation of the visual world in depth. Binocular integration occurs in layer $2 / 3$ in the primary visual cortex (V1), where inhibitory lateral connections control monocular inputs from the thalamorecipient layer 4

\footnotetext{
Received Oct. 14, 2019; revised May 12, 2020; accepted May 14, 2020.

Author contributions: Y.S., E.V., and R.F.H. designed research; Y.S. and E.V. performed research; P.R.-N. contributed unpublished reagents/analytic tools; Y.S. analyzed data; Y.S., R.F.H., and E.V. wrote the paper.

This work was supported by Canadian Institutes of Health Research Grants 148579 (to R.F.H.) and MOP111003 (to E.V.), Natural Sciences and Engineering Research Council of Canada Grants 228103 (to R.F.H.) and 238835-2011 (to E.V.), and the Foundation Nazareth and Louis Braille Institute (INLB, Longueuil, Canada; E.V.). We thank the (enter for Interdisciplinary Research in Rehabilitation of Greater Montreal and the Fonds de la Recherche du Québec en Santé (FRQS) Vision Health Research Network for their resource support and to Jacqueline Higgins for her assistance with data collection.

${ }^{*}$ R.F.H. and E.V. contributed equally to this work.

The authors declare no competing financial interests.

Correspondence should be addressed to Robert F. Hess at robert.hess@mcgill.ca or Elvire Vaucher at elvire.vaucher@umontreal.ca.

https://doi.org/10.1523/JNEUROSCI.2484-19.2020

Copyright $(2020$ the authors
}

(Başgöze et al., 2018). Previous work has implicated the endogenous neurotransmitter acetylcholine (ACh) in the excitatory/inhibitory $(\mathrm{E} / \mathrm{I})$ balance of $\mathrm{V} 1$, modulating the gain of thalamocortical synapses in layer $4 \mathrm{c}$ while also inhibiting intracortical interactions (Disney et al., 2012; Obermayer et al., 2017). Here, we use binocular rivalry, a sensitive probe of interocular dynamics (Tong et al., 2006), to characterize ACh's role in binocular integration. Importantly, mixed visibility during rivalry highlights periods when complete interocular suppression fails. On the contrary, exclusive visibility indicates instances of complete perceptual suppression, recently causally linked to enhanced GABAergic inhibition (Mentch et al., 2019). Consequently, the diverse phenomenology of binocular rivalry percepts constitutes an indirect assay of cortical E/I balance (Robertson et al., 2013, 2016; Van Loon et al., 2013; Mentch et al., 2019).

Using a double-blind placebo-controlled crossover design, we demonstrate that a single administration of the acetlycholinesterase inhibitor (AChEI) donepezil (5 mg, oral) strongly affects binocular rivalry dynamics, with important perceptual consequences. Cholinergic stimulation via donepezil enhances both the fraction and median duration of mixed visibility during rivalry, thereby reducing the amount of time one eye suppresses the other. 
Simultaneously, ACh also reduces the rate of rivalry, another sensitive probe of cortical E/I balance (Robertson et al., 2013; Van Loon et al., 2013). Our findings indicate that ACh plays a fundamental role in modulating binocular vision, providing new insights into the neurophysiological basis of human binocularity and on ACh's role in visual perception.

\section{Materials and Methods}

A total of 23 individuals completed two experimental sessions where binocular rivalry measurements were obtained before and after taking either donepezil or a placebo (lactose) pill. The binocular rivalry task consisted of a dichoptic stimulus where participants viewed a left-tilted grating in one eye and a right-tilted grating in the other for $90 \mathrm{~s}$, continuously indicating via key-press whether they were seeing (1) the left eye's image, (2) the right eye's image, (3) a piecemeal mixture of the two images, or (4) a superimposed mixture of the two images (Fig. 1). We used this task to better characterize the mixed percepts while also encouraging participants not to miscategorize a mixed percept as exclusive.

Subjects

A total of 24 individuals enrolled in the study. One participant was excluded from the study due to a failure to complete the full experiment, therefore in sum, 23 individuals participated the study (13 males; age: $25 \pm 3$; range: $20-32$ ).

All subjects met the inclusion criteria (non-smoker, normal or corrected-to-normal visual acuity, normal stereo vision, no history of any neurologic or ocular diseases, no prescription medications). The bodymass-index range was specified as $17-26 \mathrm{~kg} / \mathrm{m}^{2}$ to ensure a similar distribution of the drug across subjects. All subjects were naive to the purpose of the experiment. A standard clinical and neurologic examination was performed by Pedro Rosa-Neto, MD, Ph.D. (Douglas Hospital, McGill University). In addition, a stereoacuity test and an ECG recording were performed before the beginning of the experiment. Subjects were monitored for their safety during the experimental sessions with several blood pressure measurements taken.

Subjects gave written informed consent before the experiment. Data were collected and kept secure in the laboratory of E.V. Participants were enrolled by the student researcher Y.S., and their random allocation sequence was conducted by E.V. by assigning drug/placebo in numbered containers. Subjects received financial compensation to cover travel expenses and time spent participating in the experiment at a rate of $\$ 15 /$ $\mathrm{h}$. The procedures were in accordance with the Helsinki Declaration of 2013 and the ethical standards of the Comité d'éthique de la recherche en santé, Université de Montréal, approval \#12-084-CERES-P.

\section{Apparatus}

Each session took place in a quiet room with dim light. Visual stimuli were generated and controlled by an Apple MacBook Pro 2008 computer (MacOSX) running MATLAB R2012B (MathWorks, RRID:SCR_001622) with the Psychophysics toolbox (Pelli, 1997; Brainard, 1997) RRID:SCR_ 002881. Stimuli were presented on a $\gamma$-corrected cathode ray tube monitor (LG) driven at a resolution of $1024 \times 768$ pixels, with a refresh rate of $75 \mathrm{~Hz}$ and a measured mean luminance of $60 \mathrm{~cd} / \mathrm{m}^{-2}$. Participants viewed stimuli through an eight-mirror modified Wheatstone stereoscope so that the left image was only seen by the left eye and the right image by the right eye. The position of the participant's head was stabilized with a chin rest at a viewing distance of $57 \mathrm{~cm}$.

\section{Donepezil pharmacological enhancement}

Donepezil is a reversible, non-competitive, highly selective AChEI with a half-life of $80 \mathrm{~h}$ and a peak plasma level of $4.1 \pm 1.5 \mathrm{~h}$ after intake (Rogers et al., 1998); $5 \mathrm{mg}$ of donepezil is the lowest prescribed dose which induces beneficial cognitive effects with very low adverse reaction incidence (Prvulovic and Schneider, 2014; Kang et al., 2014) and has produced several reported effects on adult vision (Silver et al., 2008; Rokem and Silver, 2010, 2013; Chamoun et al., 2017; Gratton et al., 2017). Importantly, although higher doses of donepezil may yield
A

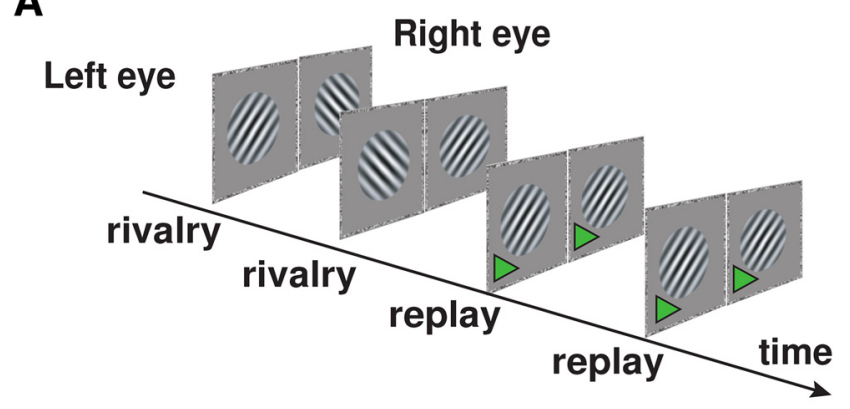

B

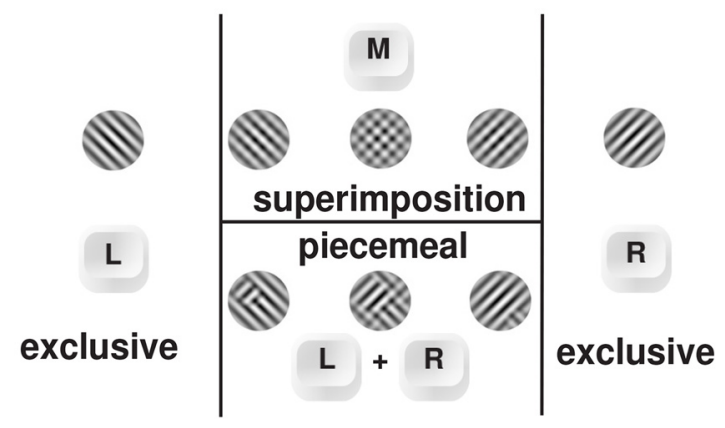

Figure 1. Experimental design. $\boldsymbol{A}$, Each block consisted of two rivalry runs (where participants viewed left-tilted and right-tilted gratings presented individually to the two eyes) and two replay runs (where participants watched computer-generated videos of simulated binocular rivalry, presented identically to both eyes), each lasting 90 s. B, Participants were instructed to continuously indicate via key-press whether they were seeing (I) the left eye's image, $(r)$ the right eye's image, $(I+r)$ a piecemeal mixture of the two images, or $(m)$ a superimposed mixture of the two images.

stronger effects on vision, a lower dose is more physiologically relevant to understanding the underlying, natural mechanisms of the visual system as it would not imbalance cortical neuromodulator levels as dramatically. Three hours before posttreatment testing, subjects ingested one cellulose capsule containing either 5-mg donepezil (auro-donepezil, Auro Pharma Inc) or lactose placebo, with water (Rokem and Silver, 2010; Chamoun et al., 2017; Sheynin et al., 2019a). The experimenter and subjects were naive to the experimental conditions.

\section{Binocular rivalry task}

We adapted a previously developed (Skerswetat et al., 2018) binocular rivalry task for our experiment to quantify the fractions and median durations of exclusive, piecemeal, superimposition, and overall mixed percepts (for illustrations, see Fig. 1B) At the beginning of each session, participants were shown images on a document that illustrated the differences between the left-oriented, right-oriented, and superimposition versus piecemeal mixed percepts. Participants were told that they would see a dynamic stimulus during the experiment and that their task was to track what they were seeing, with particular attention to timeliness and accuracy.

Participants were given the option to continuously indicate whether they were seeing either (1) an exclusively left-tilted grating, (2) an exclusively right-tilted grating, (3) a superimposition mixed percept, or (4) a piecemeal mixed percept. Participants used three adjacent keys for the task, using the left to indicate exclusive left-tilt, right for right-tilt, a holding down a combination of the left and right keys for the piecemeal percepts, and the middle key for the superimposition percepts. In our instructions, we specified that the criterion for exclusive percepts should be $\sim 90 \%$ left or right oriented.

\section{Rivalry replay control}

We used a rivalry replay control condition to characterize the criterion used for categorizing a percept as mixed and to quantify the latency of binocular rivalry responses (Robertson et al., 2013, 2016). The replay 
control consisted of computer-generated videos presented binocularly, where we oscillated the stimulus from left-oriented gratings to right-oriented gratings along a continuous scale, such that the midpoint of this oscillation would produce a complete mixture of the two gratings. Each experimental block consisted of two binocular rivalry runs followed by two rivalry replay runs. Each replay run was generated using the time series extracted from the participants' data in a preceding binocular rivalry run within the same block, replaying the participant's rivalry dynamics so as to reduce the likelihood that the participant was aware of the fact that the replay control was a different experimental condition. The initial replay runs used to confirm a participant's key-mapping were generated artificially using a square function smoothened with a Gaussian kernel ( window $=30 \mathrm{~s}$ ), where the exclusive percepts lasted exactly $2 \mathrm{~s}$ per phase and the intermediary mixed percept lasted $1 \mathrm{~s}$ per phase, and the percept oscillated evenly around a fully mixed percept. One of the replay runs in each block was generated with piecemeal mixed visibility as the mixed category, and the other with superimposition mixed visibility, so as to be able to characterize differences in criterion or response latency for these two different percept types. We extracted the average criterion used to categorize a percept as mixed by taking the mean value of the physical stimulus across all timepoints when the participant indicated they switched from exclusive to mixed visibility. The response latency was extracted by finding the time value corresponding to the minimum root mean square error (RMS) between the participant's responses and the physical stimulus. To obtain an estimate of the overall criterion and response latency for binocular rivalry (where piecemeal and superimposition both appear within a single run), we averaged the criterions and latencies across the two piecemeal and superimposition runs in each block.

\section{Experimental design and statistical analysis}

Participants (for details, see above, Subjects) were randomly allocated to either group 1 (donepezil first session, placebo second session) or group 2 (placebo first session, donepezil second session). Group assignment was counterbalanced across participants to control for possible sessionorder effects. The experimenter was not aware of the treatment condition of the two group assignments until after data collection was complete. For safety purposes, the experimenter recorded the participant's systolic blood pressure at baseline and monitored blood pressure levels throughout the experiment.

The general protocol of each experimental block is outlined in Figure $1 A$. Each block consisted of two binocular rivalry runs followed by two rivalry replay runs, each lasting $90 \mathrm{~s}$. We confirmed subjects correctly learned the key mapping corresponding to the percept categories by administering two replay runs at the beginning of every session, one run corresponding to the piecemeal mixed category, and the other to the superimposition category.

Each run (rivalry and replay) began with a dichoptic nonius cross presented inside a $3^{\circ}$ oval surrounded by a black-and-white noise ( 1 cycle/degree) frame $\left(\right.$ side $\left.=10^{\circ}\right)$. The observer was asked to make keypresses to adjust the position of the two frames to calibrate the optimal position for comfortable fusion. After confirmation, the participant was instructed to fixate at a fixation $\operatorname{dot}\left(0.2^{\circ}\right)$ and place their hands on the appropriate keys to begin responding to the rivalry task. After a keypress, the dichoptic stimulus appeared and participants began responding to what they were observing on the monitor using the keypress instructions we provided at the beginning of each session. Subsequent runs were initiated after a brief break where subjects viewed a mean-gray background screen. Subjects performed four experimental blocks before and after taking donepezil/placebo (after a 3-h drug incubation period). During the incubation period, subjects were instructed to keep both eyes open and do normal activities such as watching a movie or doing computer work in a well-lit room.

Baseline and posttreatment measurements were drawn from four experimental blocks. We implemented a mandatory 2-min break between each experimental block to prevent fatigue. The orientation of the gratings seen by the eyes during the rivalry runs was flipped between the two runs in each rivalry block to counterbalance possible orientation-eye biases and to interrupt any possible adaptation effects that would result in an increase in mixed visibility (Klink et al., 2010). We discarded the first experimental block in both baseline and posttreatment measurements to account for possible errors made in the beginning of the task.

Baseline and posttreatment measurements took place over the course of $\sim 30 \mathrm{~min}$. The half-life of donepezil is $4.1 \pm 1.5 \mathrm{~h}$ after intake. We therefore chose to begin postdeprivation testing at $3 \mathrm{~h}$ after drug administration to maximize the potency of the drug at the time of testing. During the drug incubation period, participants were instructed to keep their eyes open and do activities that require visual perception such as watching a movie, doing homework, or walking around the laboratory. Participants were also given a brief questionnaire before and after each experimental session that used a Likert scale (1-5) to quantify levels of arousal, along with two short answer questions to characterize whether they noticed any perceptual or psychosomatic differences between (1) the morning and afternoon sessions, and (2) between the two experimental sessions.

After completing the first session of an experiment, each participant was assigned a scheduled date to return for completing their second session. To ensure there was no residual effects from the previous session, all sessions were spaced at least one week apart from one another (Mean $\pm \mathrm{SD}=8 \pm 1.3 \mathrm{~d}$ ).

Using the preprocessing methodology described in detail by Sheynin et al. (2019b), we extracted key aspects of binocular rivalry dynamics corresponding to the overall fractions and median durations of (1) exclusive visibility, (2) piecemeal visibility, (3) superimposition visibility, and (4) aggregate (superimposition + piecemeal) mixed visibility. This preprocessing pipeline consisted of four stages: (1) remove the first and last percept states in the time series as well as all percept states shorter than $250 \mathrm{~ms}$ to obtain the preprocessed time series, (2) extract the distribution of percept phase durations for each state from the processed time series, (3) calculate the median and sum of these distributions to obtain the median and fraction duration of each of the states in each rivalry block. To obtain the fraction and median duration of aggregate mixed visibility, (4) we concatenated adjacent piecemeal and superimposition percepts in the original rivalry time-series data and re-executed the preprocessing paradigm mentioned above.

In addition, we extracted the overall rate of rivalry, defined as the total number of switches between the exclusive percepts divided by the run duration (Robertson et al., 2013, 2016). In order to quantify the magnitude of the effect of the two treatment conditions relative to baseline, we divided mean posttreatment values by the mean baseline values to obtain post/pre ratios for each dependent variable across both experimental conditions. Furthermore, we also evaluated the effect of donepezil treatment by comparing post/pre values for variables obtained from the replay rivalry control condition. These were the following: (1) the criterion used to categorize a percept as mixed and (2) the response latency.

To ascertain the effect of donepezil on binocular rivalry dynamics, we conducted a repeated measures MANOVA of the drug effect on the post/pre values for the seven dependent variables obtained from the binocular rivalry experiment. Additionally, we conducted a separate repeated measures MANOVA of the drug effect on the two dependent variables obtained from the replay rivalry control condition to rule out the possibility that donepezil-induced effects in our data could be attributed to factors other than changes in binocular rivalry dynamics.

When the omnibus MANOVA test was significant, we conducted post hoc Bonferroni-corrected Tukey's HSD tests on each dependent variable to determine which variables were affected by donepezil. In addition, we obtained 95\% confidence intervals (1000 bootstrapped samples, each drawing 23 subjects with replacement) for the mean drug effect for each dependent variable. All statistical analyses were conducted using IBM SPSS Statistics (version 24). This study was not preregistered. The datasets generated and analyzed during the current study are available online at https://github.com/ysheynin/Cholinergic_Modulation_Binocular_Vision.

\section{Results}

A primary aim of our study was to evaluate the effect of donepezil on the diverse phenomenology of binocular rivalry percepts. 


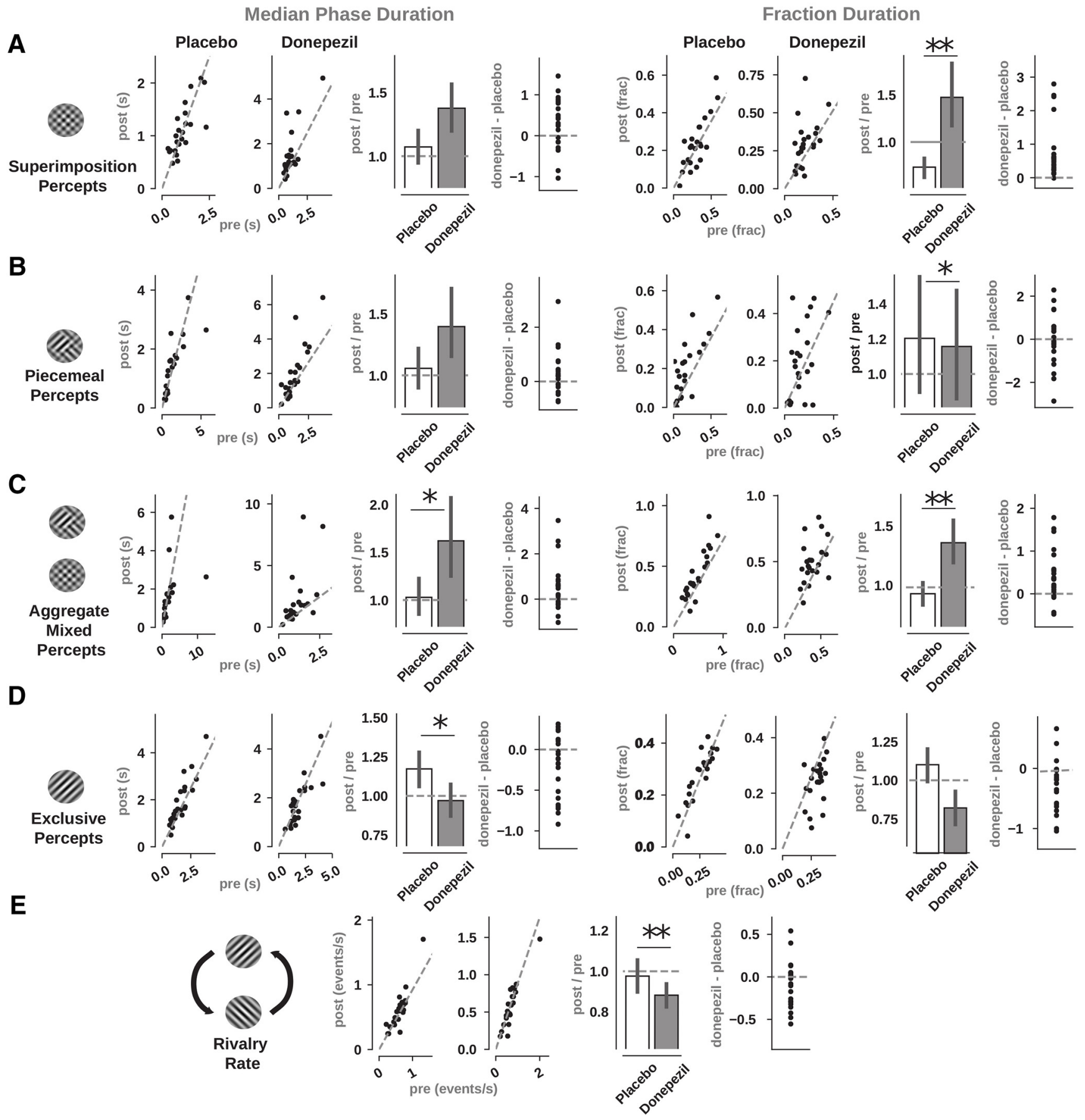

Figure 2. Effect of donepezil on binocular rivalry dynamics. Each row illustrates (1) scatter plots of the raw data observed by taking the mean of each dependent variable $(\boldsymbol{A}-\boldsymbol{E})$ across three binocular rivalry runs at baseline ( $x$-axis) and after treatment ( $y$-axis) for both the placebo and donepezil sessions; (2) a bar plot where each bar represents the average of three binocular rivalry blocks conducted $3 \mathrm{~h}$ after ingesting a donepezil/placebo pill, divided by the average of three identical rivalry blocks at baseline, averaged across participants; and (3) a scatter plot of donepezil's effect on each dependent variable obtained by subtracting the post/pre values of the placebo session from those of the donepezil session. Left column illustrates data for the median durations of the four percept types, while the right column illustrates data for the fraction durations of each percept type $(\boldsymbol{A}-\boldsymbol{D})$. Black asterisks between bars indicate significant differences observed with Tukey's HSD tests in the post/pre values between treatment conditions. Error bars are $95 \%$ confidence intervals (from 1000 bootstrapped samples) of the mean; **Bonferroni-corrected $p<0.01,{ }^{*} p<0.05$.

To that end, our task allowed us to measure the median and fraction duration of piecemeal and superimposition mixed percepts during rivalry, as well as the median and fraction duration of aggregate mixed visibility and exclusive visibility. In addition, we also examined the rate of rivalry as another dependent variable.

Figure 2 illustrates the effect of donepezil on these aspects of binocular rivalry dynamics. A repeated measures MANOVA of the effect of session on the post/pre values for these variables was significant $\left(F_{(1,22)}=2.68, p=0.04, \eta_{p}^{2}=0.63\right.$, Wilks' $\left.\lambda=0.37\right)$, indicating that donepezil significantly altered binocular rivalry dynamics when compared with the placebo control.

We observed an interesting pattern emerge within the two mixed percept subcategories. Piecemeal percepts, where the two images appear combined as in a mosaic, are proposed to emerge from a reduction of the spatial coherence of interocular inhibition, whereas superimposition percepts, where the two 


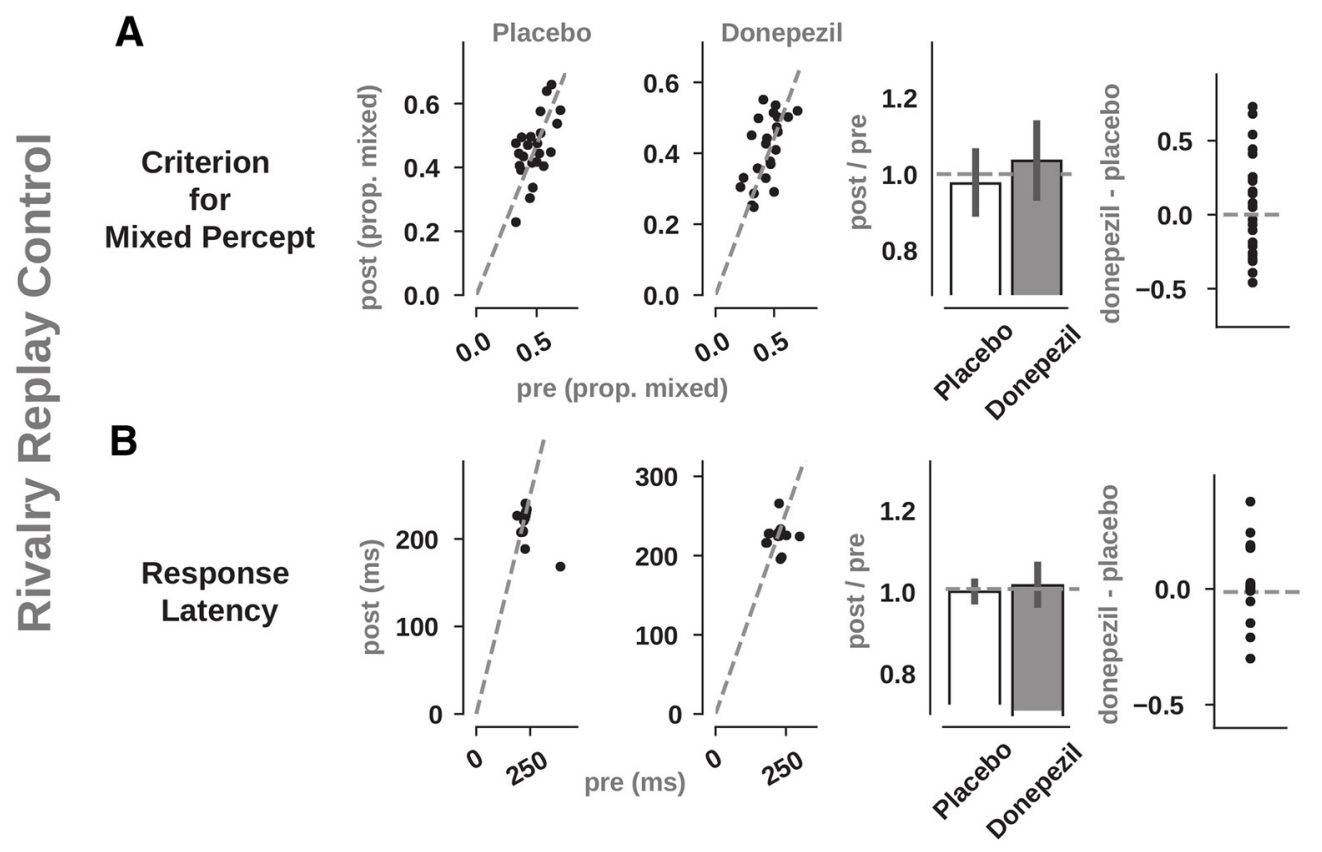

Figure 3. Effect of donepezil on replay rivalry criterion and response latency. To ensure any treatment effects were the result of changes in visual neural activity as opposed to changes in motor or executive function, we implemented a rivalry replay control experiment where participants watched computer-generated videos of simulated binocular rivalry, presented identically to both eyes. This control experiment allowed us to measure $(\boldsymbol{A})$ the criterion for categorizing a physical stimulus as "mixed" and $(\boldsymbol{B})$ the response latency for discriminating changes in the physical stimulus. There were no observable differences between donepezil and placebo sessions in the response latency or mixed criterion, suggesting that changes in non-simulated binocular rivalry dynamics are attributed to ACh-induced changes in the network dynamics of visual cortex. See Figure 2 for additional details regarding the plots.

component gratings appear overlaid as in a plaid, likely correspond to decreases in the gain of interocular inhibition (Klink et al., 2010; Fig. 1A). Cholinergic stimulation enhances the fraction visibility of superimposition percepts by $70 \%(p=0.001,95 \% \mathrm{CI}$ (Confidence Interval) [31\%, 108\%]; Fig. 2A, right), while simultaneously reducing the fraction of piecemeal percepts by $15 \%$ ( $p=0.03,95 \%$ CI [9\%, 30\%]; Fig. 2B, right). Although not statistically significant, we observed a different pattern emerge for the median durations of these percepts. Both the median duration of piecemeal $(\mathrm{M}=49 \%, p=0.06,95 \% \mathrm{CI}[-2 \%, 81 \%])$ and superimposition $(\mathrm{M}=54 \%, p=0.06,95 \%$ CI $[-3 \%, 110 \%])$ percepts increased with administration of donepezil. Despite this inconsistency (which may be due to variance in the baseline data between the two sessions), these data suggest that ACh predominantly modulates the overall gain of interocular inhibition and reduces the spatial coherence of the inhibition.

Furthermore, our results indicate that donepezil significantly enhances the fraction of aggregate mixed visibility during rivalry by $43 \%$ ( $p=0.002,95 \%$ CI [ $18 \%, 68 \%]$; Fig. $2 C$, right $)$ and likewise increases the median duration of mixed visibility by $20 \%$ $(p=0.025,95 \%$ CI [4\%, 37\%]; Fig. $2 C$, left). Increases in the fraction of mixed visibility were observed in 19 out of 23 participants, where eight of these individuals exhibited donepezilinduced increases of $>50 \%$. These changes were reciprocated in measures of exclusive visibility during rivalry, where donepezil reduces both the median duration of exclusive percepts by $78 \%$ $(p=0.01,95 \%$ CI $[18 \%, 139 \%]$; Fig. $2 D$, left $)$ and the fraction of exclusive percepts by 59\% ( $p=0.46,95 \%$ CI $[-220 \%, 103 \%]$; Fig. $2 D$, right). The rate of binocular rivalry also decreased by $36 \%$ $(p=0.002,95 \%$ CI $[13 \%, 54 \%]$; Fig. $2 E)$ in the donepezil condition relative to placebo, suggesting attenuation of cortical inhibition (Robertson et al., 2013; Van Loon et al., 2013; Mentch et al., 2019). Together, these results point to an ACh-induced increase in the visibility of mixed percepts during rivalry, likely due to a shift in favor of excitation.
Despite the dramatic increase in the visibility of mixed percepts, these changes were not reflected in individuals' self-report of their experience after the experiment, nor during a control condition where we generated rivalry playback videos and measured the criterion used for categorizing a percept as mixed (for details, see Materials and Methods). While mixed visibility increased substantially on donepezil, a MANOVA conducted on the criterion and response latency from the rivalry replay control experiment was not significant $\left(F_{(1,22)}=1.4, p=0.24, n_{p}^{2}=0.12\right.$, Wilks' $\lambda=0.88$ ), indicating that the perceptual changes we observe in the donepezil condition cannot be caused by changes in subjects' response criteria (Fig. $3 A$ ) or response latency (Fig. $3 B$ ) and can only be attributed to changes in neural activity.

\section{Discussion}

Together, these results point to an ACh-induced increase in the visibility of mixed percepts during rivalry, especially superimposed percepts, and likely due to a shift in favor of excitatory drive. Importantly, our findings cannot be attributed solely to an increase in the gain of purely monocular signals, as previous work has demonstrated that enhanced stimulus contrast, which increases monocular gain, actually reduces mixed visibility during rivalry (Hollins, 1980). Our results are therefore aligned with the notion that cholinergic potentiation reduces the gain of interocular inhibition in layers $2 / 3$ of V1.

Likewise, our results align themselves with recent evidence published in this journal (Mentch et al., 2019), which implicated GABAergic inhibition in gating perceptual awareness during binocular rivalry. While enhanced GABAergic transmission increases perceptual suppression and occasionally increases rivalry alternation rates, our study demonstrated, for the first time, that enhanced cholinergic activity reduces perceptual exclusivity during rivalry, decreases alternation rates, and thus has an opposite effect to GABA. In fact, our data demonstrate that 
superimposition, not piecemeal percepts, is maximally enhanced by donepezil. This is consistent with the idea that interocular inhibition is reduced. Moreover, the reduced rate of rivalry marked by increases in mixed visibility, as found in our study, points to an excitation-dominant cortical response profile, opposed to an increased switch rate with increased GABAergic transmission. Combined, these findings lend additional support for previous models of binocular rivalry that implicated changes in E/I balance in binocular cortex to perceptual awareness during rivalry (Wilson, 2003; Brascamp et al., 2013; Said and Heeger, 2013).

Critically, our findings implicate the cholinergic system in modulating the E/I balance of binocular visual cortex. Cholinergic fibers modulate various inhibitory circuits: feed-forward inhibition, lateral inhibition, and disinhibition (Obermayer et al., 2017), so we cannot directly infer the effect on GABAergic circuits induced by enhanced cholinergic transmission from our experiment. However, it is highly plausible, given our results, recent evidence from Mentch et al. (2019), as well as previous electrophysiological work from Krueger and Disney (2019), that the predominant cholinergic enhancement effect in V1 would reduce the inhibitory drive that enables ocular suppression, leading to increases in interocular interactions and the occurrence of mixed percepts.

Future work will reveal the extent to which specific aspects of binocular vision, such as stereopsis or summation, can be shaped by $\mathrm{ACh}$, as well as whether cholinergic stimulation can be used therapeutically for disorders of binocular function.

\section{References}

Başgöze Z, Mackey AP, Cooper EA (2018) Plasticity and adaptation in adult binocular vision. Curr Biol 28:r1406-r1413.

Brainard DH (1997) The psychophysics toolbox. Spat Vis 10:433-436.

Brascamp J, Sohn H, Lee SH, Blake R (2013) A monocular contribution to stimulus rivalry. Proc Natl Acad Sci USA 110:8337-8344.

Chamoun M, Huppé-Gourgues F, Legault I, Rosa-Neto P, Dumbrava D, Faubert J, Vaucher E (2017) Cholinergic potentiation improves perceptual-cognitive training of healthy young adults in three dimensional multiple object tracking. Front Hum Neurosci 11:128-129.

Disney AA, Aoki C, Hawken MJ (2012) Cholinergic suppression of visual responses in primate $\mathrm{V} 1$ is mediated by GABAergic inhibition. J Neurophysiol 108:1907-1923.

Gratton C, Yousef S, Aarts E, Wallace DL, D’Esposito M, Silver MA (2017) Cholinergic, but not dopaminergic or noradrenergic, enhancement sharpens visual spatial perception in humans. J Neurosci 37:4405-4415.

Hollins M (1980) The effect of contrast on the completeness of binocular rivalry suppression. Percept Psychophys 27:550-556.
Kang JI, Huppé-Gourgues F, Vaucher E (2014) Boosting visual cortex function and plasticity with acetylcholine to enhance visual perception. Front Syst Neurosci 8:172.

Klink PC, Brascamp JW, Blake R, Van Wezel RJA (2010) Experience-driven plasticity in binocular vision. Curr Biol 20:1464-1469.

Krueger J, Disney AA (2019) Structure and function of dual-source cholinergic modulation in early vision. J Comp Neurol 527:738-750.

Mentch J, Spiegel A, Ricciardi C, Robertson CE (2019) GABAergic inhibition gates perceptual awareness during binocular rivalry. J Neurosci 39:83988407.

Obermayer J, Verhoog MB, Luchicchi A, Mansvelder HD (2017) Cholinergic modulation of cortical microcircuits is layer-specific: evidence from rodent, monkey and human brain. Front Neural Circuits 11:100.

Pelli DG (1997) Pixel independence: measuring spatial interactions on a CRT display. Spat Vis 10:443-446.

Prvulovic D, Schneider B (2014) Pharmacokinetic and pharmacodynamic evaluation of donepezil for the treatment of Alzheimer's disease. Expert Opin Drug Metab Toxicol 10:1039-1050.

Robertson CE, Kravitz DJ, Freyberg J, Baron-Cohen S, Baker CI (2013) Slower rate of binocular rivalry in autism. J Neurosci 33:16983-16991.

Robertson CE, Ratai EM, Kanwisher N (2016) Reduced GABAergic action in the autistic brain. Curr Biol 26:80-85.

Rogers C, Sukovaty P, Lee F, Rogers SL, Cooper NM, Sukovaty R, Pederson JE, Lee JN, Friedhoff LT, Rogers C, Sukovaty P, Lee F (1998) Pharmacokinetic and pharmacodynamic profile of donepezil $\mathrm{HCl}$ following multiple oral doses. Br J Clin Pharmacol 46:7-12.

Rokem A, Silver MA (2010) Cholinergic enhancement augments magnitude and specificity of visual perceptual learning in healthy humans. Curr Biol 20:1723-1728

Rokem A, Silver MA (2013) The benefits of cholinergic enhancement during perceptual learning are long-lasting. Front Comput Neurosci 7:66.

Said CP, Heeger DJ (2013) A model of binocular rivalry and cross-orientation suppression. PLoS Comput Biol 9:e1002991.

Sheynin Y, Chamoun M, Baldwin AS, Rosa-Neto P, Hess RF, Vaucher E (2019a) Cholinergic potentiation alters perceptual eye dominance plasticity induced by a few hours of monocular patching in adults. Front Neurosci 13:22-12.

Sheynin Y, Proulx S, Hess RF (2019b) Temporary monocular occlusion facilitates binocular fusion during rivalry. J Vis 19:23-17.

Silver MA, Shenhav A, D'Esposito M, Esposito MD, D'Esposito M (2008) Cholinergic enhancement reduces spatial spread of visual responses in human early visual cortex. Neuron 60:904-914.

Skerswetat J, Formankiewicz MA, Waugh SJ (2018) More superimposition for contrast-modulated than luminance-modulated stimuli during binocular rivalry. Vision Res 142:40-51.

Tong F, Meng M, Blake R (2006) Neural bases of binocular rivalry. Trends Cogn Sci 10:502-511.

Van Loon AM, Knapen T, Scholte HS, St. John-Saaltink E, Donner TH, Lamme VAF (2013) GABA shapes the dynamics of bistable perception. Curr Biol 23:823-827.

Wilson HR (2003) Computational evidence for a rivalry hierarchy in vision. Proc Natl Acad Sci USA 100:14499-14503. 